\title{
CMV viral load in bronchoalveolar lavage for diagnosis of pneumonia in allogeneic hematopoietic stem cell transplantation
}

\author{
L Iglesias $^{1}$, MM Perera ${ }^{2}$, L Torres-Miñana ${ }^{2}$ and MJ Pena-López ${ }^{1}$
}

The objective of this report is to analyze the value of CMV viral load (VL) in bronchoalveolar lavage (BAL) in recipients of allogeneic hematopoietic stem cell transplantation (AHSCT) and to analyze the concordance between CMV quantification in plasma and BAL samples. Fifty-six patients were included; in 16 (28.6\%) patients with symptoms of lung disease a BAL sample was collected, $7(43.7 \%)$ patients had a VL $>150$ copies/mL (six had probable CMV pneumonia). Patients with CMV pneumonia were female (100\%), of median age 53.5 years, and the median time from transplantion to onset of symptoms was 67.5 days. Pneumonia was early in three cases (incidence 5.4\%). Median BAL VL was 53250 copies/mL and plasma VL was 538 copies/mL (in one patient, the plasma VL was negative). Three (50.0\%) patients had an improvement in the first week of treatment, and plasma VL became negative; the other $3(50.0 \%)$ patients died with a CMV pneumonia refractory to treatment. Therefore, any value of CMV VL in BAL, especially if it is greater than plasma, with compatible signs or symptoms, should be considered suggestive of CMV pneumonia in recipients of AHSCT and they should receive specific treatment.

Bone Marrow Transplantation (2017) 52, 895-897; doi:10.1038/bmt.2017.11; published online 20 February 2017

\section{INTRODUCTION}

CMV causes severe pneumonia after allogeneic hematopoietic stem cell transplantation (AHSCT), with a high mortality rate in spite of prevention strategies. ${ }^{1}$ The diagnosis of pneumonia traditionally includes the detection in bronchoalveolar lavage (BAL) or lung tissue samples by virus isolation, histopathologic testing, immunohistochemical analysis or in situ hybridization. ${ }^{2,3}$ The viral isolation has poor clinical utility because it has low sensitivity and reproducibility and is slow. ${ }^{4-6}$ Quantification of viral load (VL) in BAL specimens by real-time PCR to diagnose CMV pneumonia is not evaluated sufficiently. ${ }^{7-9}$ To determine the value of CMV VL in BAL in patients who have received an AHSCT with pneumonia, we performed a study of all patients with positive VL in BAL who were followed for at least 100 days after AHSCT.

\section{SUBJECTS AND METHODS}

From January 2013 to December 2015, 69 patients received an AHSCT in our hospital; 56 patients were included in the study and 13 were excluded because they died before day +100 of causes unrelated to CMV infection. In patients, who had symptoms of lung disease, a BAL specimen was collected by bronchoscopy and the following tests were done: cultures for bacteria, fungi, mycobacteria and viruses; PCR assays for respiratory syncytial virus, influenza $A$ and $B$, metapneumovirus, parainfluenza 1-4, rhinovirus, adenovirus and coronavirus (PneumoVir, Genomica, Madrid, Spain); and detection of Pneumocystis jirovecii antigen by indirect immunofluorescence (Monofluo kit P. jirovecii, Bio Rad, Marnes-la-Coquette, France). Quantification of CMV DNA in BAL and plasma samples, obtained simultaneously, was performed using COBAS Ampliprep/Cobas Taqman CMV System (Roche, Mannheim, Germany) according to the manufacturer's specifications.

We reviewed the medical records of patients in whom a VL of CMV in BAL was positive.

To describe patients' characteristics, we used percentage, median and range. The study was approved by the Ethics Committee of our hospital.

\section{Definitions}

CMV pneumonia was classified into proven and probable as defined by Ljungman et al. ${ }^{3}$

Early and late pneumonia. Pneumonia before or after 100 days of transplant, respectively.

Refractory infection to treatment. Persistence of viraemia after 2 weeks of specific antiviral treatment.

CMV-attributable mortality. Death within 6 months after onset of symptoms, with CMV disease, with no other cause directly contributing to death.

\section{RESULTS}

Out of 56 patients included, 31 (55.4\%) were male and the median age was 51 years (range, 17-68). The median follow-up time was 473 days (range, 53-1231). Thirty-five (62.5\%) patients had a

\footnotetext{
${ }^{1}$ Microbiology Service, University Hospital of Gran Canaria Dr Negrín, Las Palmas, Spain and ${ }^{2}$ Hematology Service, University Hospital of Gran Canaria Dr Negrín, Las Palmas, Spain. Correspondence: Dr L Iglesias-Llorente, Microbiology Service, University Hospital of Gran Canaria Dr Negrín, Barranco de la Ballena s/n, Las Palmas de Gran Canaria, Las Palmas 35019, Spain.

E-mail: laura.igll@hotmail.com

Received 7 December 2016; revised 20 December 2016; accepted 4 January 2017; published online 20 February 2017
} 


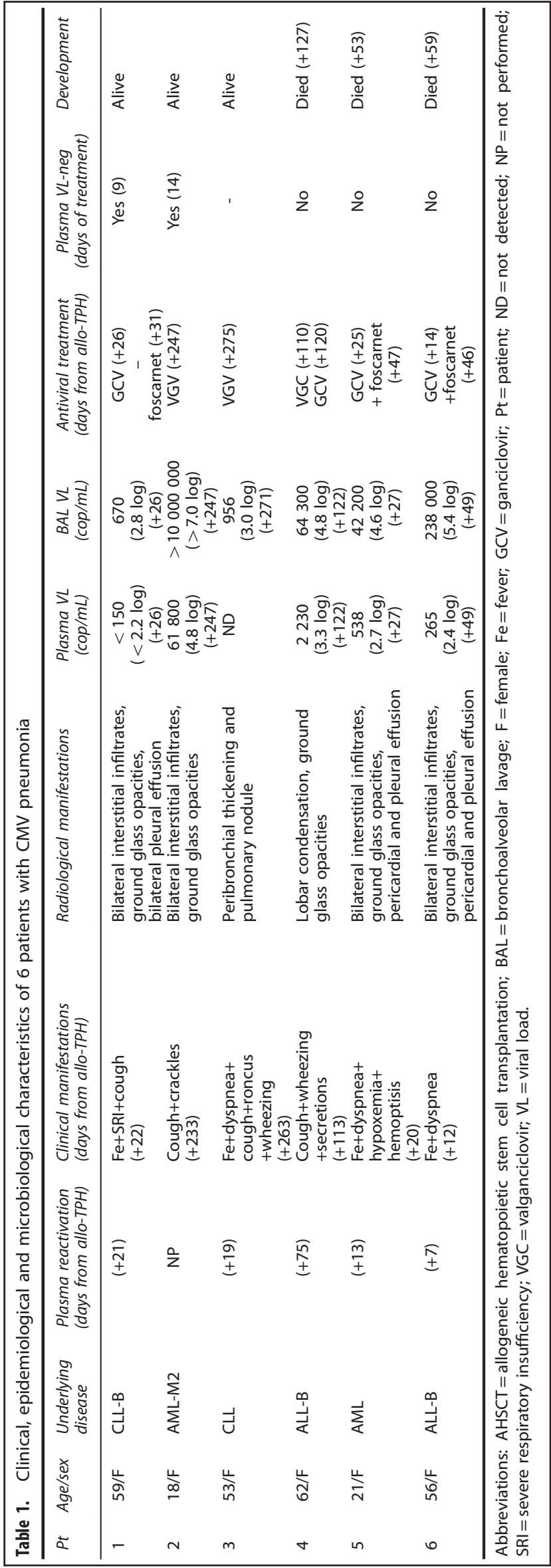

plasma CMV reactivation in the first 100 days after transplantation. In $16(28.6 \%)$ patients a BAL sample was collected, and the VL of CMV in BAL was positive in 10 patients (in $7>150$ copies $/ \mathrm{mL}$ and in $3>150$ copies $/ \mathrm{mL}$ ), negative in 2 and in 4 patients with negative virologic culture, the sample were insufficient for VL. Three patients with VL less than 150 copies/mL were diagnosed with idiopathic pneumonia, but they had a good evolution with ganciclovir treatment. Of the 7 patients with BAL VL higher than 150 copies $/ \mathrm{mL}, 6(85.7 \%)$ were diagnosed of probable CMV pneumonia and in 1 (14.3\%) case CMV disease was discounted. This patient presented on day +7 post-transplant with respiratory symptoms, bilateral interstitial infiltrate and pleural effusion, and the VL was undetectable in plasma and BAL specimens. On day +21 post transplant, a plasma viremia $(<150$ copies $/ \mathrm{mL}$ ) was detected, and ganciclovir treatment was started on day +35 because the patient had a clinical improvement but no radiological improvement; plasma VL was 265 copies $/ \mathrm{mL}$ and BAL VL was 321 copies/mL. Plasma and BAL VL became undetectable without complete clinical or radiological improvement and died on day +165 post transplant by alveolar hemorrhage associated with bronchiolitis obliterans in the context of a chronic GvHD and tumor progression.

Table 1 shows the clinical, epidemiological and microbiological characteristics of the six patients with CMV pneumonia $10.7 \%$ of all patients). All patients were female and median age was 53.5 years old (range 18-61). The median time from transplantation to onset of symptoms was 67.5 (range 12-263) days. Pneumonia was presented early in three cases (incidence 5.4\%) and late in other three. The median BAL VL was 53250 (4.7 log) copies/mL (range $670->10000000)$. In five cases plasma VL was positive, with a median of 538 (2.7 log) copies/mL (range $<150-61800$ ). All patients had a plasma VL less than BAL VL, and in one case plasma VL was negative. Only one BAL sample was positive for CMV by virological culture, with a VL $>10000000$ copies $/ \mathrm{mL}$. In none of the cases was another pathogen detected.

All patients were treated with valganciclovir or ganciclovir; three of them were added or changed to foscarnet; two patients because of clinical deterioration and detectable plasma VL at 22 and 32 days after initiation of therapy (cases 5 and 6) and one because of adverse events (case 1). Only one patient received early therapy (case 4). Three (50.0\%) patients had a clinical and radiological improvement in the first 7 days following the start of the treatment, with a reduction of plasma VL to undetectable. Three $(50.0 \%)$ patients died with a diagnosis of CMV pneumonia refractory to treatment at 7,28 and 45 days after the start of treatment (5.4\% of AHSTC patients).

\section{DISCUSSION}

CMV pneumonia in AHSCT remains a common complication with high mortality even with specific treatment. ${ }^{1,4}$ Diagnostic tests with high sensitivity and speed, such as BAL VL, can be useful to identify patients in the early stage of infection or patients at risk of developing pneumonia, but it has not been validated for diagnosis of disease. ${ }^{6,10}$

As far as we know, this is the first study to evaluate the role of $\mathrm{VL}$ in $\mathrm{BAL}$ in the diagnosis of pneumonia regarding clinical data in patients who have received an AHSCT and to analyze the concordance between CMV quantitation in plasma and BAL specimens. Studies have analyzed the value of VL in BAL in other patient populations, mainly in lung-transplanted patients, in which it is considered that high VL (above 46000 copies $/ \mathrm{mL}$ or 500000 copies $/ \mathrm{mL}$ ) is suggestive of CMV pneumonia. ${ }^{11-13}$ In our study, the patients with probable CMV pneumonia had a wide range $(670->10000000$ copies $/ \mathrm{mL})$ of $\mathrm{VL}$ and a median above 50000 copies/mL. However, all patients with a BAL VL less than 1000 copies $/ \mathrm{mL}$ (including three patients with loads $<150$ copies $/ \mathrm{mL}$ ) responded to treatment with ganciclovir and had a good 
evolution, which could be related to an early stage of the disease. Moreover, $75 \%$ of patients with VL higher than 50000 copies $/ \mathrm{mL}$, died of refractory pneumonia. The non-response to treatment in patients with high VL could be related to an advanced clinical stage or resistance to ganciclovir. All patients' plasma VL was lower than in BAL; one patient even had an undetectable plasma VL. This fact was described by other authors, ${ }^{11,13,14}$ which could imply that plasma VL monitoring has limitations to anticipate the development of pneumonia, and therefore low or undetectable viremia levels did not exclude CMV pneumonia. In the only patient with detectable BAL VL, without CMV pneumonia, the BAL VL was not higher than in plasma.

In this study, $10.7 \%$ of patients who received an AHSCT were diagnosed with probable CMV pneumonia with a high percentage of mortality (50\%) with a specific treatment. As described in other studies, ${ }^{15}$ the factor associated with increased mortality was the persistent CMV DNAemia for more than 2 weeks in spite of antiviral therapy, which could be related to ganciclovir resistance, although we could not analyze this fact. In two patients who died, the treatment was changed to foscarnet very late (after 20 days of ganciclovir treatment, the plasma VL did not become negative).

In our study, virological culture performance was very low and only a sample of BAL with $\mathrm{VL}>10$ million copies $/ \mathrm{mL}$ was positive. This could be because patients had already begun antiviral treatment before samples were taken, or because of the lower performance of cell culture when VL was low.

In conclusion, based on our results, any value of CMV VL in BAL, especially if it is greater than in plasma, could be considered suggestive of CMV pneumonia in patients who receive AHSCT; such patients should receive specific treatment, because without specific treatment the mortality could be very high. However, further studies assessing BAL VL in patients who recieve an AHSCT are necessary. We believe that this study, in spite of the low number of patients, is important because there are no publications in which BAL CMV VL was evaluated in patients with AHSCT. It is noted that conventional diagnosis methods in BAL and the plasma VL are limited and have a low performance. Tests to quantify VL of CMV are available in many clinical microbiology laboratories and this is an advantage because they could be used to quantify the BAL VL samples. These results should not be extrapolated to other populations, such as HIV or solid organ transplant recipients.

\section{CONFLICT OF INTEREST}

The authors declare no conflict of interest.

\section{REFERENCES}

1 Erard V, Guthrie KA, Seo S, Smith J, Huang M, Chien J et al. Reduced mortality of cytomegalovirus pneumonia after hematopoietic cell transplantation due to antiviral therapy and changes in transplantation practices. Clin Infect Dis 2015; 61: 31-39.

2 Ljungman P, Griffiths P, Paya C. Definitions of Cytomegalovirus infection and disease in transplant recipients. Clin Infect Dis 2002; 34: 1094-1097.

3 Ljungman P, Boeckh M, Hirsch HH, Josephson F, Lundgren J, Nichols $G$ et al. Definitions of CMV infection and disease in transplant patiens for use in clinical trials. Clin Infect Dis 2016; 64: 87-91.

4 Travi G, Pergam SA. Cytomegalovirus pneumonia in hematopoietic stem cell recipients. J Intensive Care Med 2014; 29: 200-212.

5 Chemaly RF, Yen-Lieberman B, Castilla EA, Reilly A, Arrigain S, Farver C et al. Correlation between viral loads of cytomegalovirus in blood and bronchoalveolar lavage specimens from lung transplant recipients determined by histology and immunohistochemistry. J Clin Microbiol 2004; 42: 2168-2172.

6 Seo S, Renaud C, Kuypers JM, Chiu CY, Huang ML, Samayoa E et al. Idiopathic pneumonia syndrome after hematopoietic cell transplantation: evidence of occult infectious etiologies. Blood 2015; 125: 3789-3797.

7 Cathomas BG, Morris P, Pekle P, Cunningham I, Emanuel D. Rapid diagnosis of cytomegalovirus pneumonia in marrow transplant recipients by bronchoalveolar lavage using the polymerase chain reaction, virus culture, and the direct immunostaining of alveolar cells. Blood 1993; 81: 1909-1914.

8 Eriksson BM, Brytting M, Zweygberg-Wirgart B, Hillerdal G, Olding-Stenkvist E, Linde A. Diagnosis of cytomegalovirus in bronchoalveolar lavage by polymerase chain reaction, in comparison with virus isolation and detection of viral antigen. Scand J Infect Dis 1993; 25: 421-427.

9 Tan SK, Burgener EB, Waggoner JJ, Gajurel K, Gonzalez S, Chen SF et al. Molecular and culture-based bronchoalveolar lavage fluid testing for the diagnosis of cytomegalovirus penumonitis. Open Forum Infect Dis 2016; 3: ofv212.

10 Emery V, Zuckerman M, Jackson G, Aitken C, Osman H, Pagliuca A et al. Management of cytomegalovirus infection in haemopoietic stem cell transplantation. Br J Haematol 2013; 162: 25-39.

11 Chemaly RF, Yen-Lieberman B, Chapman J, Reilly A, Bekele BN, Gordon SM et al. Clinical utility of cytomegalovirus viral load in bronchoalveolar lavage in lung transplant recipients. Am J Transplant 2005; 5: 544-548.

12 Henke-Gendo C, Ganzenmueller T, Kluba J, Harste G, Raggub L, Heim A. Improved quantitative PCR protocols for adenovirus and CMV with an internal inhibition control system and automated nucleic acid isolation. J Med Virol 2012; 84: 890-896.

13 Westall GP, Michaelides A, Williams TJ, Snell Gl, Kotsimbos TC. Human cytomegalovirus load in plasma and bronchoalveolar lavage fluid: a longitudinal study of lung transplant recipients. J Infect Dis 2004; 190: 1076-1083.

14 Ruell J, Barnes C, Mutton K, Foulkes B, Chang J, Cavet J et al. Active CMV disease does not always correlate with viral load detection. Bone Marrow Transplant 2007; 40: 55-61.

15 Liu J, Kong J, Chang YJ, Chen H, Chen YH, Han W et al. Patients with refractory cytomegalovirus (CMV) infection following allogeneic haematopoietic stem cell transplantation are at high risk for CMV disease and non-relapse mortality. Clin Microbiol Infect 2015; 21: 1121, e9-e15. 